
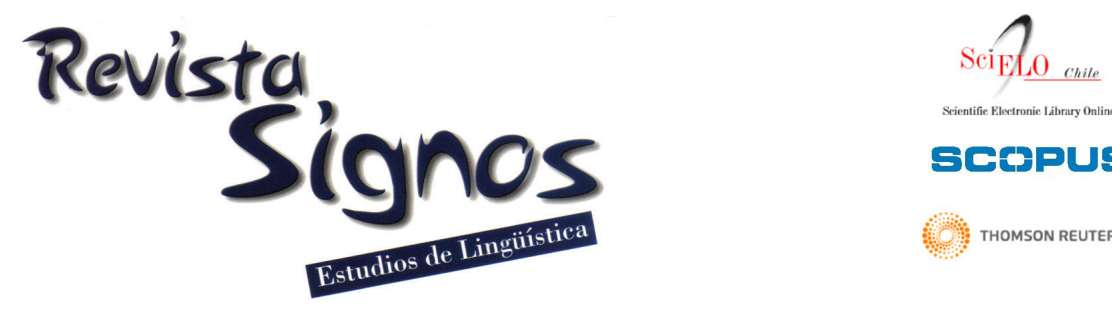

\title{
Principios, competencia y anulamientos en el orden de constituyentes oracionales*
}

\section{Principles, competition and annulments in the order of sentence constituents}

\author{
Claudio Pinuer \\ cpinuer@udec.cl \\ Universidad de Concepción \\ Chile
}

\author{
Teresa Oteíza \\ moteizas@uc.cl \\ Pontificia Universidad de Católica de Chile \\ Chile
}

Recibido: 20-XII-20I0 / Aceptado: I9-VIII-20I I

\begin{abstract}
Resumen: En este trabajo presentamos la revisión de 12 principios de orden oracional de amplia ubicuidad disciplinaria, los cuales se exploran en sus propiedades gramaticales, informativas y cognitivas; y a continuación se sistematizan en una tabla de atributos lineares formada por los rasgos más representativos de cada nivel. Los principios son examinados a partir del patrón SVO en la oración di/transitiva, en la cual se describen los parámetros que especifican la posición de los complementos argumentales de acuerdo con su forma de des/activación. El estudio ha permitido verificar que los principios actúan según elecciones informativas, propiedades lexicogramaticales y requerimientos cognitivos. Se puede destacar que el orden de constituyentes oracionales es resultado de la interacción entre los parámetros, en un proceso de complementación y anulamiento recíproco.
\end{abstract}

Palabras Clave: Orden de palabras, linearización, SVO, sintaxis. 


\begin{abstract}
In this article we present a revision of the 12 principles of sentence order of great disciplinary ubiquity, which are explored regarding their grammatical, informative and cognitive properties. These principles are systematized in a table of linear attributes formed by the most representative features of each level. The principles are analysed from a SVO pattern in the di/transitive sentence, in which the parameters that specify the position of argumental complements according to its de/activation type are described. The study has allowed us to verify that the principles apply according to the informative choices, grammatical properties and cognitive requirements. It is possible to highlight that the order of sentence constituents is the result of the interaction between the parameters in a process of reciprocal complementarity and annulment.
\end{abstract}

Key Words: Word order, linearization, SVO, syntax.

\section{INTRODUCCIÓN}

El estudio del orden oracional dispone de una larga trayectoria en el ámbito lingüístico, aunque en buena parte de ella ocupó un lugar secundario, entre los fenómenos de "sintaxis figurada" (RAE, 2009: 2970), realidad que cambiaría diametralmente, sobre todo en el siglo $X X^{\prime}$. Desde este periodo, el orden se ha investigado con interés exponencial en sus alcances semántico (marco argumental del predicado), sintáctico (complejidad de constituyentes), acústico (asignación del acento oracional), tipológico (jerarquía SVO), pragmático (estrategia informativa) y cognitivo (costo de procesamiento). En mayor o menor grado, cada una de las nociones recogidas en esta apretada compilación se ha explorado sobre la base de fundamentos epistemológicos que pueden llegar a ser antagónicos, si se tiene en cuenta que los paradigmas generativo (Bosque \& Gutiérrez-Rexach, 2009) y funcional (Dik, 1997) han explicado el orden a través de reglas de concordancia y colocación, respectivamente.

La extensa y multidisciplinaria atención concedida al fenómeno linear ha redundado en una profusa investigación, cuyos desiguales planteamientos hacen imposible armonizar bajo un solo enfoque o disciplina los hallazgos obtenidos dentro y fuera de la lingüística. La consecuencia más importante de esto es que cualquier estudio sobre orden oracional que pretenda alcanzar adecuación empírica -y este se propone lograrlo-, debe incluir nociones provenientes de diversas disciplinas, elaboradas incluso en marcos epistemológicos discordantes, como los que sustentan generativismo y funcionalismo. Con todo, nuestra perspectiva teórica matriz será funcional, pues adoptamos la teoría multifuncional del orden de Dik (1997) como modelo explicativo central y definimos la lengua como sistema sociosemiótico (Halliday, I978, 2005).
Pondremos en discusión varias propuestas en el bien entendido de que por separado cada una captura aspectos diferentes del mismo fenómeno y a través del conjunto se accede a una comprensión más amplia y, lo que puede ser más importante, por vía de explicitar la forma de interacción entre esas heterogéneas capturas. En consonancia con lo anterior, el objetivo fundamental que persigue el presente estudio es exponer un modelo teórico sobre los principios lineares de alta ubicuidad epistemológica y disciplinaria, a través del análisis de su papel en el orden de la oración declarativa di/ transitiva en español.

Para intentar construir un modelo que junto con captar rasgos críticos del encadenamiento oracional devele su forma de interacción, las tareas centrales consistirán en: (i) revisar articuladamente principios singularizados en su origen disciplinar y empírico; (ii) sistematizar sus propiedades fundamentales; $y$ (iii) explicar su funcionamiento según opciones de activación (empatía), complementación (integridad de dominio $\leftrightarrow$ estabilidad funcional) y anulamiento (complejidad $\leftrightarrows$ animacidad).

En el estudio de casos se tomará como punto de partida el denominado orden canónico, que corresponde al trazado sintáctico SVO, cuya jerarquía lo posiciona como el lugar más idóneo desde el cual abordar la indagación del fenómeno distribucional. Precisamente, en la primera sección analizaremos dicho taxema de acuerdo con su valor tipológico y como tópico privilegiado de la teoría lingüística y disciplinas afines, cuyos principales hallazgos han revitalizado la investigación del orden de constituyentes. La segunda sección estará dedicada a revisar los componentes de la teoría multifuncional del orden de Dik (1997) que 
sobresalen por su capacidad explicativa, y también a examinar otras nociones (principios) que han potenciado la explicación del fenómeno linear. En la sección tercera y final propondremos una sistematización de los principios y profundizaremos en los efectos de su des/activación en la fisonomía sintagmática de la oración declarativa.

\section{La preeminencia SVO}

\section{I.I. Fundamentos teóricos y tipológicos}

Los trabajos comparativos de Greenberg (1963) afianzaron el orden oracional como uno de los principales parámetros tipológicos. El llamado principio greenberiano estableció seis patrones distribucionales: SVO, SOV, VSO, VOS, OVS, OSV; entre los cuales, SVO fue jerarquizado como la opción de mayor prominencia. Revisaremos a continuación algunos fundamentos de tal jerarquía, necesarios para interpretar adecuadamente este genuino dogma lingüístico.

En primer lugar, destacaremos que SVO es el patrón sintáctico no-marcado de las oraciones declarativas, transitivas (I) y ditransitivas (2):

(I) Los vecinos apelaron la resolución del concejo municipal.

(2) Los vecinos presentaron la apelación al concejo municipal.

No estará demás advertir que este ordenamiento puede ser anulado por la asignación de relieve informativo, una de cuyas manifestaciones es la ubicación de un funtivo 'inoportuno' en una posición extrema (zona de alta sensibilidad pragmática), fenómeno que se ilustra enseguida con un caso de dislocación (3) y otro de anteposición focal (4):

(3) 'La resolución del concejo municipal' apelaron los vecinos.

(4) $\left[\mathrm{Al}\right.$ concejo municipal $\left.{ }_{\mathrm{Foco}}\right]$ presentaron los vecinos la apelación.

Dada la falta de acuerdo, conviene precisar cómo ha de entenderse la relación $(I) /(3)$ y $(2) /(4)$. Para el funcionalismo, (3) y (4) no son derivaciones ni modificaciones lineales de las correspondientes no marcadas (I) y (2). Aceptar una o ambas posibilidades conlleva admitir hipótesis generativas como reglas de ascensión (dislocación a la izquierda) y de movimiento (anteposición focal), soluciones incompatibles con una perspectiva funcionalista, donde no se distingue entre estructura superficial y estructura profunda (Butler, Mairal, Martín \& Ruiz, 1999). Digamos, abreviadamente, que las cuatro oraciones son magnitudes autónomas y representan elecciones disponibles en el sistema lexicogramatical.

Por otra parte, SVO es caracterizado como orden central e interpretado como el trazado sintáctico de menor constreñimiento y disponible por ello para un número amplio de contextos, propiedades verificables en su compatibilidad con los modus declarativo (en las 4 oraciones anteriores), interrogativo (5) y exclamativo (6):

(5) ¿Los vecinos apelaron la resolución del concejo municipal?

(6) ¡Los vecinos apelaron la resolución del concejo municipal!

Un aspecto interesante de plantear es que la condición de rasgo central no depende del factor cuantitativo en el sentido de no ser por obligación la propiedad que exhiba el máximo de apariciones, lo que fuerza a distinguir entre orden básico y frecuente. Si bien en español coinciden ambas formas, conviene hacer algún comentario sobre esta distinción.

El estatus nuclear de un rasgo lingüístico se relaciona, por una parte, con la ya reseñada flexibilidad contextual y, por otra, con la capacidad de expresar en grado óptimo un atributo tipológico distintivo con independencia a la cuantía de realizaciones. Por ejemplo, en el ámbito de la oración declarativa di/transitiva, la prenuclearización de los objetos inducida por la integración pronominal (El Ministro entregará en estos días nueva propuesta a los estudiantes $\rightarrow$ El Ministros se las entregará en estos días) no pone en duda la jerarquía SVO, que sigue conservando su estatus de realización central.

La posición matriz de SVO es concomitante con la universalidad del modus declarativo y dado que las modalidades interrogativa y exclamativa son también centrales, su carácter marcado se deduce de la mayor rentabilidad de aquel. Recordemos la afinidad de su patrón sintáctico con la interrogación y exclamación. 
Si bien no cabe impugnar el mayor estándar de la aserción, juzgamos necesario precisar el lugar ocupado por SVO en dicha modalidad, a fin de prevenir generalizaciones infundadas. En concreto, dentro de las realizaciones declarativas existen alternativas de orden neutro, de lo cual se infiere que una organización diferente a SVO no implica por sí sola una distribución marcada como ocurre en las siguientes oraciones, cuyo patrón linear no modifica la canonicidad de la estructura informativa:

(7) Se le olvidó ‘su clave secreta'.

(8) Se conocieron 'nuevos detalles del caso judicial'.

(9) A los políticos no les agradan 'las comisiones fiscalizadoras'.

(I0) Aparecieron 'andinistas extraviados en la precordillera'.

En estos casos, la ubicación final del sujeto es propia de construcciones singularizadas por su naturaleza reflexiva (7), pasiva pseudorrefleja (8), inacusativa (9) y presentativa (10), todas las cuales, como ha destacado Siewierska (Martínez, 1999), tienen una organización informativa no-marcada pues se inician con material dado y finalizan con material nuevo. $\mathrm{Si}$ bien cada oración presenta un orden constreñido, el componente informativo no muestra la misma condición, de tal forma que la canonicidad de una oración declarativa no dependerá solo de la presencia de SVO.

Según precisa Dik (1997), la taxonomía greenberiana ha recibido varias modificaciones. Por ejemplo, Lehmann (1973) y Vennemann (1974) readecuaron los seis patrones en dos taxemas, $\mathrm{VO} / \mathrm{OV}$, capturando la distribución núcleo-modificador. En un cambio más radical, Hengeveld, Rijkhoff y Siewierska (2004) adoptaron el parámetro predicación inicial, media y final, debido a la imposibilidad de identificar funciones sintácticas (sujeto, objeto) y categorías léxicas (verbo) en lenguas de elasticidad funcional, caso del samoano, donde:

"categorization of full words is not given a priori in the lexicon. It is only their actual occurrence in a particular environment which gives them the status of a verb or a noun" (Hengeveld et al., 2004: 540).

Por su parte, Hengeveld y Mackenzie (2008) aplicaron la distinción lenguas tópico-orientadas/lenguas focoorientadas, que representa un giro en los parámetros predominantes de los estudios tipológicos, por lo general, gramaticales. En efecto, ambas nociones se sustentan en la organización informativa, sobre cuya base las lenguas pueden dividirse entre aquellas que privilegian la ubicación inicial de información compartida (tópico-orientadas) y aquellas que sitúan en esa posición la información de mayor pertinencia comunicativa, conocida o no (focoorientadas). Según Alonso-Cortés (2008: 339), estas lenguas tienen una orientación pragmática pues "sus argumentos están alineados de acuerdo con su estatuto en el curso de la información".

Para finalizar, mencionaremos el parámetro lenguas sujeto-orientadas/lenguas tópico-orientadas, dentro del cual el español es clasificado como sujetoorientada, no obstante su capacidad para elidir (en cláusulas reducidas) o anular (en oraciones impersonales) la función. Con este, los anteriores $y$ otros criterios formulados sobre principios nomorfosintácticos se supera la limitación que trae el empleo de variables carentes de generalización (como la categoría verbo y la función sujeto).

Debido a la búsqueda de consistencia translingüística sabemos hoy que todas las lenguas disponen de la organización tópico/comento, pero no todas distribuyen el material oracional de acuerdo con las funciones formales sujeto y predicado. A modo de corolario, diremos junto con Padilla (2005: 26) que:

“las necesidades pragmáticas pueden ser universales, pero los mecanismos que codifican las lenguas no tienen por qué coincidir”.

Las indagaciones orientadas por las nuevas propuestas tipológicas no prescinden de parámetros gramaticales, aunque su empleo conlleve el riesgo de las generalizaciones tipológicas carentes de sustento lexicogramatical (como la universalización del patrón SVO), las cuales, por supuesto, no pueden ser descartadas si solo se tiene en cuenta que la mayor parte de las lenguas son aún desconocidas en el sentido técnico de la palabra.

Como cabe esperar, los criterios de amplitud descriptiva priorizan el plano semántico (por ejemplo, \pm animacidad) e informativo (por ejemplo, tópico/comento) por no entrar en conflicto con la forma en que las lenguas delimitan su sistema morfosintáctico. En todo caso, la aplicación de nociones uniformes no logra establecer conexiones tipológicas pormenorizadas, motivo por el cual es 
común el empleo conjunto de parámetros diversos, estrategia que analizaremos más adelante cuando se examinen los datos registrados en la Tabla $\mathrm{I}$.

Aun cuando este trabajo tiene al funcionalismo como modelo teórico central, no es posible ignorar el planteamiento generativo, pues algunas de sus nociones explicativas del orden de constituyentes se emplean en la actualidad con gran difusión, varias de ellas surgidas en los desarrollos posteriores al denominado generativismo clásico. Para comenzar, recordemos que en su etapa fundacional se postuló que la estructura profunda disponía de un orden lineal y que las variaciones sintagmáticas eran fenómenos de superficie, determinados por aspectos retóricos de la actuación.

En la teoría de rección y ligamiento, desarrollada en la década de los ' 80 , el enfoque generativo distinguió entre lenguas configuracionales y lenguas no-configuracionales sobre la base de un orden relativamente fijo en las primeras y relativamente libre en las segundas. Según Eguren y Fernández (2004: I5I), la determinación del orden lineal del núcleo y sus complementos se explicita:

"por medio de la fijación de un parámetro, el Parámetro de la Posición del Núcleo, que le permite al hablante optar entre colocar el núcleo antes o después de sus complementos. [De acuerdo con este Parámetro] el español es una «lengua de núcleo inicial» (los complementos se sitúan a la derecha del núcleo en nuestra lengua)”.

En el marco del programa minimista, la oración, precisan Bosque y Gutiérrez-Rexach (2009), es concebida como un objeto sintáctico complejo derivado (en la estructura profunda) de un proceso de fusión (emerge), el cual especifica la posición de los constituyentes de acuerdo con las propiedades de cada pieza. De este modo, la ubicación de una palabra es parte de su manifestación como categoría lingüística, a la vez que expresión del cotejo de sus rasgos.

Debido a que el cotejo de rasgos (feature checking) genera construcciones, es crítico que sus elementos concuerden correctamente sus propiedades. Por ejemplo, en la oración 'Los problemas crean las teorías', la concordancia de persona y número y la ubicación asignan al primer sintagma la función de sujeto, en tanto que la posición ( $y$ no la concordancia flexiva) establece la función OD para la segunda frase. Este chequeo de propiedades morfosintácticas corresponde a un caso de 'cotejo de rasgos semánticamente interpretables' por el efecto en el significado de la estructura, entre los cuales, conviene insistir por el propósito de este artículo, se encuentra el orden de constituyentes.

Los roles sintácticos conllevan entonces la posición estructural como manifestación de los rasgos de las piezas léxicas seleccionadas para cumplir una función específica. Ahora bien, cuando los sintagmas no ocupan su posición estructural o canónica, como en el caso de dislocación (ejemplo 3), el minimalismo sintáctico formula una estructura más alta al sintagma flexión (el cual deriva la oración), tesis que para nuestro trabajo tiene el interés especial de excluir el desplazamiento como hipótesis explicativa.

Por contrapartida, la anteposición focal (ejemplo 4) es generada por una operación de movimiento (move), etiquetada con el nombre de S(intagma) Foco, la cual especifica que:

"los elementos focalizados se desplazan a la proyección de foco para cotejar el rasgo de foco en aplicación al criterio de igual nombre" (Bosque \& Gutiérrez-Rexach, 2009: 697).

El movimiento se activa en casos muy puntuales, hecho que lleva a Bosque y Gutiérrez-Rexach (2009) a explicar este fenómeno a través del principio de avidez (greed) formulado por Chomsky, el cual predice que:

"un objeto sintáctico se desplazará solamente para cotejar sus propios rasgos. Así pues, el movimiento está determinado por la necesidad de cotejar un rasgo propio, y no los rasgos de otros objetos sintácticos existentes o potenciales" (Bosque \& Gutiérrez-Rexach, 2009: 223).

Las operaciones asociadas al cotejo de rasgos no son artificios descriptivos, sino formulaciones que pretenden capturar conocimiento lingüístico natural. En este sentido, no corresponden a abstracciones tradicionales (a pesar del uso de nomenclatura común como sintagma), sino nociones intuitivas que subyacen a la generación de entidades lingüísticas por lo cual son apropiadas como hipótesis explicativas desde un punto de vista teórico. 
Buena parte del planteamiento minimista se resume en el axioma léxico, de gran circulación en la gramática española actual, que asigna al predicado la capacidad de especificar la posición estructural de los argumentos (Rodríguez, 2005), pero esta hipótesis, además de limitarse al orden canónico, deja fuera los parámetros extralingüísticos (como iconicidad y prominencia informativa) que regulan el orden del enunciado y son evidencia de la actividad comunicativa del hablante. Como esperamos demostrar con la siguiente cita, una caracterización fidedigna del lenguaje no puede pasar por alto su papel en la realidad extralingüística:

"One of the basic functions of language is to segment the flux of happenings in the world into units which speakers refer to events" (Rappaport, Doron \& Sichel, 20I0: I).

A pesar de la magnitud de sus diferencias, generativismo y funcionalismo (tipológico) coinciden en asunciones importantes, entre ellas, la valoración de parámetros formulados con independencia a generalizaciones lingüísticas tradicionales y la imposibilidad de que una noción formal, como SVO, pueda tener mayor poder explicativo que una noción cognitiva. Con todo, el referido taxema alcanza amplia cobertura translingüística y ofrece una fructifera complementación con otros parámetros, según veremos enseguida, dejando hasta aquí esta breve y puntual referencia a la teoría generativa sin no antes reiterar que en el marco del desarrollo vigente del minimalismo, la dislocación y la anteposición focal son explicadas sintácticamente a través del cotejo de rasgos semánticamente interpretables².

Según Hengeveld et al. (2004), el año 1987 se comprobó que el $75 \%$ de las lenguas investigadas hasta ese periodo tenía SVO como orden central, preeminencia verificada en estudios tipológicos posteriores que refrendaron el hallazgo en un marco descriptivo más amplio, donde el orden se complementa con otros parámetros de naturaleza informativa y semántica. Una lograda muestra de esta diversificación es el trabajo de Heine y König (2008) sobre la posición de objetos con verbos ditransitivos, de cuyos resultados generales solo podremos comentar unos cuantos datos que confirman la potestad SVO, en línea con otros parámetros, como se observa en la Tabla I.

Primeramente, se ratifica la centralidad del patrón SVO en español, para el cual se establece un ordenamiento IOC (construcción de objeto indirecto) que la ubica entre las lenguas que marcan con idéntico procedimiento el argumento tema (Theme) de los verbos di/transitivos y el argumento paciente de los verbos monotransitivos. En el caso del español, esta marca es cero $(\varnothing)$, debido a que ambos argumentos se realizan como objeto directo, rol sintáctico que selecciona la marca casual cuando la frase denota una entidad supra/humana - personificada, que ciertos principios, como la definitud que ilustramos en (I I), tienen la capacidad de cancelar:

\section{(II) Juan busca (a) un socio para crear una empresa.}

Además, el español es situado entre las lenguas de disposición $\mathbf{T}$ (heme) - $\mathbf{R}$ (ecipient), dada su tendencia a priorizar el OD por sobre el OI (a la inversa del inglés), con autonomía al rasgo de animacidad que con mucha regularidad presenta el segundo objeto. Según Heine y König (2008: 3) el orden estructural R-T es más central debido a que: "is moore frequent, particularly with higher positions of the animacy/ defitiness scale."

Por último, W(eight), complejidad creciente en Dik (1997), predice la ubicación final del complemento más pesado por su complejidad sintáctica (relativa, por ejemplo, a longitud) y/o mayor densidad léxica, expresada en el volumen de significación y de carga presuposicional. Este principio explica que los

Tabla I. Parámetros prevalentes de orden en la oración ditransitiva.

\begin{tabular}{|l|l|l|l|l|l|l|}
\hline Language & Family & Spoken (main country) & Word Order & Alignment & Order Structure & Principle \\
\hline Español & IE Romance & España & SVO & IOC & T-R & W \\
\hline Mapuche & Sur Andina & Chile & SVO & DOC & T - R & Viol. \\
\hline Papiamento & CP & Curazao & SVO & DOC & R-T & A \\
\hline
\end{tabular}


sintagmas con subordinación oracional tengan en el final de enunciado su posición no-marcada.

Por otra parte, nos pareció interesante reproducir la caracterización del mapuche, pues comprueba la validez intertipológica de los parámetros, capaces de vincular una lengua aglutinante como el mapuche, con una lengua flexiva como el español. Si bien coinciden en dos propiedades centrales (el orden oracional y la disposición argumental), también difieren en un rasgo muy singularizador en el mapudungun: ser individualizada como una lengua violadora de principios (Viol.), lo cual, en palabras simples, significa que en su configuración linear no subyacen los principios de mayor jerarquía translingüística, como el peso (W). Además, se distingue por su carácter de lengua DOC (double object construction), rasgo que implica (al igual que en el papiamento) identificar $\mathbf{T}, \mathbf{P}$ y $\mathbf{R}$ con idéntica marca.

Por último, la caracterización de una lengua 'nueva' como el criollo portugués $(\mathrm{CP})$ refuerza la generalidad transligüística de los rasgos tipológicos, que podrían tener la condición de 'fósiles' lingüísticos, como el patrón SVO, especializado en gramaticalizar la jerarquía del agente (Casado, Martín-Loeches, Muñoz \& Fernández-Frías, 2005), propiedad que no puede ser solo resultado del azar evolutivo.

A pesar del limitado repaso al alcance de los parámetros tipológicos, resulta de todas formas suficiente para apoyar la jerarquía de SVO como orden no-marcado, a juzgar por los datos, desde los mismos orígenes de las lenguas. Complementariamente, muchas lenguas han adoptado esa configuración tras abandonar otros esquemas configurativos, en tanto que otras avanzan en la misma dirección (Padilla, 200I).

\section{I.2. La jerarquía psicolingüística de SVO}

El patrón linear SVO ha demostrado tener prioridad cognitiva en consideración a su temprana manifestación (LI) o a su mayor facilidad de adquisición (L2). Además, se ha establecido que configura la estructura oracional más sencilla desde un punto de vista cognitivo-perceptivo (Padilla, 200 I) por ubicarse entre los enunciados que se reconocen y procesan más fácilmente (Sánchez, 2004).
En particular, la posición inicial del sujeto, establecida como zona informativa común, ha sido corrientemente interpretada como factor de enlace discursivo, que obedece a un principio psicolingüístico general que predice la simplicidad del proceso decodificador cuando el intercambio lingüístico evoluciona de la información compartida a la información que se introduce por primera vez.

La posición inicial, llamada también punto de arranque, ha recibido atención preferente en la investigación cognitiva y lingüística que ha hecho manifiesta su jerarquía e importancia en ambos campos disciplinarios. Se entiende, de acuerdo con lo anterior, que el inicio sea la zona más sensible de un enunciado a la activación de estrategias psicolingüísticas (como la mención temprana de los referentes animados) y pragmáticas (como la colocación preliminar de la información compartida), lo contrario, como advierte Lambrecht (1998: 20I), iría en sentido opuesto a la lógica:

"Given that sentence-initial position is cognitively speaking an eminently salient position, it would be a priori surprising if the prominence associated with this position could only be exploited for a single function, such as the marking of the topic relation."

Para Godeo (2000), la conexión entre la configuración sintáctica de la oración y las tareas psicolingüísticas asociadas a su procesamiento, es evidencia poderosa de la intervención del orden de palabras en el proceso de control de la atención, dentro de la cual especifica la cantidad de atención requerida para procesar la información introducida para incrementar el conocimiento del destinatario. Asimismo, Godeo (2000) valida el supuesto de que la tarea de decodificación del oyente es intrínsecamente más difícil que la operación codificadora del hablante, pues aquel desconoce lo que se va a decir. En consecuencia, el miembro no-marcado de una clase, en nuestro caso SVO, es más fácil de procesar y está orientado al receptor, mientras que el miembro marcado (por ejemplo, OVS) se dirige al hablante que, como sabe lo que va a transmitir, puede seleccionar una alternativa al orden canónico para cumplir un fin comunicativo puntual, entre ellos, el cambio de tópico discursivo.

Godeo (2000) asegura que la presentación del material lingüístico de fácil acceso debe preceder a la información menos accesible, fenómeno que nos sirve para explicar el hecho común de que 
la información compartida preceda con regular frecuencia a la información focal. Según la autora, existe una relación de correspondencia entre la capacidad limitada de la memoria de corto plazo y las configuraciones SVO y SOV, cuyo procesamiento cognitivo es menos costoso y por ello más funcional para ese tipo de memoria.

Por su parte, Branigan, Pickering y Tanaka (2008) pesquisaron el grado de influencia del principio de animacidad en la asignación de los papeles sintácticos y su distribución en enunciados oracionales. Basados en la hipótesis de que las entidades animadas poseen mayor accesibilidad y recuperabilidad, comprobaron que:

"language production is incremental, easily accessed information is processed first; animate entities therefore tend to be privileged during syntactic processes of production." (Branigan et al., 2008: 172).

Al examinar la incidencia específica del 'animado primero' en la fisonomía sintáctica, Barnigan et al. (2008: I72) concluyeron que:

"experimental cross-linguistic evidence supports that animacy simultaneously influence both grammatical function assignment and the determination of word order".

En particular, este dato explica la prioridad distribucional que recibe el sujeto agentivo.

Por último, los mismos autores citan estudios desarrollados por Prat-Sala y Branigan en los cuales estos últimos verificaron que:

"participants in a Spanish picture-description task showed a greater tendency to produce animate entities than inanimate entities as both subjects in passive sentences [ $\mathrm{La}$ mujer fue atropellada por el tren] and direct objects in left-dislocated object sentences [A la mujer la atropelló el tren]" (Branigan et al., 2008: 180).

Ambas disposiciones lineares coinciden en ubicar la entidad animada en primera posición.

Recapitulemos sobre la jerarquía SVO con los argumentos examinados y algún otro complementario:
I) Neutralidad pragmática: es el orden neutro para todas las oraciones di/transitivas y para una parte de las intransitivas.

2) Menor marcación formal: es, como consecuencia de lo anterior, el orden menos marcado para las declarativas y el que menor carga presuposicional tiene.

3) Función desambiguadora: es el orden elegido en casos de ambigüedad en la definición del sujeto de la oración (El auto chocó el camión).

4) Frecuencia: más del $90 \%$ de oraciones transitivas con frases nominales plenas en posición de sujeto y de objeto son de orden SVO (Padilla, 200I).

5) Preeminencia cognitiva: adquisición temprana (LI), mayor facilidad de aprendizaje (L2), incidencia positiva en tareas de procesamiento.

6) Predominante en las frases hechas (El frío cala los huesos).

Es comprensible, aunque no estemos necesariamente dispuestos a compartirlo, que SVO haya sido caracterizado como 'fósil sintáctico', a modo de un vestigio de etapas remotas de la evolución lingüística. También se ha señalado que la referida distribución se comporta como un principio de pregnancia, una especie de orden superior que mueve las lenguas hacia la iconicidad, la uniformidad y la economía.

\section{La materialización del orden oracional y su teorización explicativa}

\section{I. La multifuncionalidad dikeana}

Para efectos de la distribución 'seleccionada' en una oración, los principios de orden son reglas de colocación (placement rules) antes que reglas de movimiento (movement rules) que trasladan un elemento de una posición especificada a otra, según aclara Dik (1997). Detengámonos por un momento en esta afirmación:

(12) El empresario ofreció un bono de término de conflicto a los trabajadores.

(I3) Un bono de término de conflicto ofreció el empresario a los trabajadores.

(14) A los trabajadores el empresario les ofreció un bono de término de conflicto.

La oración (I2) posee el patrón SVO que, por su carácter central y ubicuo, tiende a ser interpretado como el orden sobre el cual se producirían las modificaciones distribucionales (I3) y (I4). 
Tal como señalamos con anterioridad, estos casos de alotaxis o allosentences (Lambrecht, 1998) deben entenderse como realizaciones lexicogramaticales independientes que, si bien significan lo mismo (una entidad agentiva transfiere un objeto a una entidad receptiva), no informan de igual manera (determinación de lo dado y lo nuevo).

A continuación, nos referiremos a los parámetros de orden propuestos por Dik (I997: 392-394), comenzando por aquellos principios generales (GP) que han influenciado decisivamente los estudios sobre fisonomía linear:

(GPI) The Principle of Iconic Ordering. Constituents conform to (GPI) when their ordering in one way or another iconically reflects the semantic content of the expression in which they occur.

(GP4) The Principle of Domain Integrity. Constituents prefer to remain within their proper domain;domains prefer not to be interrupted by constituents from other domains.

(GP6) The Principle of Functional Stability. Constituents with the same functional specification are preferably placed in the same position.

(GP7) The Principle of Pragmatic Highlighting. Constituents with special pragmatic functionality (New Topic, Given Topic, Completive Focus, Contrastive Focus) are preferably placed in 'special positions', including, at least, the clause-initial position.

En primer lugar, cada principio recoge un valor prominente y explicita un modo de organización. Si su formulación es correcta, tendrán la capacidad de predecir la posición de un elemento y explicar la configuración de la magnitud en la que aparece. Adicionalmente, tendrán el potencial analítico de develar la empatía establecida por el hablante con el o los factores de orden.

Por ejemplo, la oración (12) presenta el evento de acuerdo con la progresión que tendría en la realidad (GPI), esto es, desde el causante de la acción hacia el destinatario del proceso, situando el objeto como instancia de intercambio. Además, la colocación $\mathrm{V}$ OD se realiza en posición contigua, en consonancia con (GP4) que predice la realización inmediata del núcleo con su complemento más integrado. (GP6) se puede visualizar en la ubicación de los objetos dentro del dominio estructural del predicado. La activación de estos principios produce la fisonomía neutra o no-marcada de (I2), en la cual sobresale la asignación sobre un mismo $\mathrm{SN}$ de las funciones sujeto/agente/tema.

En lo que respecta a (I3) y (I4), su ordenamiento permite detectar la subyacencia de GP7, pues ambas oraciones sitúan en posición no-canónica el OD y Ol, cancelando el orden icónico y la estabilidad funcional. Para explicar su diferenciación específica, es clave el principio de animacidad, que veremos luego, de acuerdo con el cual una entidad animada tiene prioridad sobre una entidad no-animada, lo que se produce en (I4) y no en (I3).

Como se puede apreciar, el orden es regulado por un conjunto de principios, entre los cuales se establece una jerarquía que determina la estructura final del enunciado. Lo anterior permite sospechar que los principios no se despliegan en forma unilateral y que, muy por el contrario, pueden interactuar bajo ciertos niveles de tensión (Padilla, 2005) creada por las operaciones informativas del enunciatario. Estas ideas más otras certeras intuiciones sustentan la teoría multifuncional del orden elaborada por Dik (1997: 396), en la cual las nociones de interacción y tensión forman parte de las asunciones $(A)$ centrales:

(AI) The actual constituent ordering patterns found in a language are the resultant of a number of interacting principles.

(A6) Shifts in the relative force of the different principles may lead to (sometimes radical) changes in constituent ordering.

(A7) Where such changes relieve tension with respect to one principle, they may create new tension with respect to another.

Además, Dik (1997) establece que los principios son parámetros naturales, motivados informativamente, de modo que su activación no depende de pautas unívocas de manifestación. Esto explica que la primacía de (GPI) tenga mucho que ver en SVO, pero muy poco o nada en OVS, donde la intervención de (GP7) resulta clave cuando la oración no está constreñida semánticamente, fenómeno presentado en (7), (8), (9) y (I0).

Sin duda que el orden es un fenómeno gramatical, cuya explicación dista de una solución aislada o dependiente solo de factores estructurales y etimológicos. Con indudable razón, Dik (1997: 396) concluye que: "(A8) There is consequently no optimal, stable solution to the constituent ordering problem". 


\subsection{El factor cognitivo y lexicogramatical}

Existen otros principios explicativos que complementan los examinados hasta ahora (animacidad respecto de iconicidad) o los amplían con parámetros que captan otros elementos (referencialidad) del fenómeno linear. Seguidamente, examinaremos algunos de los más indagados en la investigación actual, favorecidos por permitir un mayor acceso a propiedades reveladoras del funcionamiento de la memoria y del sistema lingüístico.

La importancia superlativa asignada por las neurociencias a la posición inicial se expresa en el principio TFP (Theme First Principle), el cual establece que la información conocida/compartida/vieja se sitúa primero. Godeo (2000) relaciona este postulado cognitivo con la simplicidad del procesamiento del lenguaje que privilegia la presentación temprana de la información común, que debe permanecer en la memoria de trabajo para interpretar la información que se introduce por primera vez. La priorización del principio TFP es señal de empatía del hablante con el interlocutor, pues la organización informativa plasmada en su mensaje busca facilitar la comprensión en el destinatario.

Por otra parte, el principio dikeano de integridad de dominio (GP4) se complementa con el principio VOB (Verb Object Bonding), según el cual el objeto de un verbo di/transitivo forma con el signo verbal un vínculo más cohesivo sintáctica y semánticamente que el establecido entre verbo y sujeto, por lo que en condiciones de predicación no-marcada aparecerán en forma contigua. El principio VOB es apoyado por planteamientos composicionales, entre ellos que: "el verbo y sus complementos forman un constituyente que deja fuera al sujeto" (Escandell, 2004: I 42). Es indudable la analogía que existe entre este parámetro y la hipótesis de cotejo de rasgos semánticamente interpretables.

Otro principio que ha atraído el interés de los psicolingüistas (Branigan et al., 2008), es la noción AFP (Animated First Principle) que establece la prioridad de una magnitud de referencia animada para ocupar la primera posición de acuerdo con una jerarquía interna donde sobresale lo más humano: animado humano $>$ animado no humano $>$ no animado humano > no animado no humano. Esta escala predice que, incluso entre referentes inanimados, el rasgo de animacidad concederá precedencia a unidades lingüísticas de contenido personificado del tipo 'el gobierno' (no animado humano).
En coincidencia con el principio AFP, el principio de Persona establece, según Heine y König (2008), una gradación entre los pronombre personales: los referentes activos (Yo-Tú) tienen primacía sobre la llamada no-persona (Él), que corresponde a la entidad extradialógica, no necesariamente animada. Este principio también empalma con el postulado de iconicidad pues los sujetos dialógicos son participantes activos y, como tal, su identificación priorizada refleja más auténticamente el acontecimiento codificado en la oración.

Similar proximidad presenta el parámetro Tematicidad, relacionado con la visibilización de las entidades según el grado de control que ejercen en el desarrollo de un acontecimiento. La tematicidad establece que los participantes activos tienen mayor relieve por ser quienes hacen posible el evento y por ello tienen preferencia para ocupar la posición inicial de la oración.

Un principio menos divulgado es el axioma de Definitud o Referencialidad, el cual predice que entre las diversas categorías lingüísticas, aquella(s) de mayor aptitud para denotar una entidad con el mínimo de incertidumbre mostrará(n) tendencia a ocupar una posición prominente, de forma que en toda lengua que disponga de ítemes léxicos para denotar entidades (humanas o no), aquellos con función 'etiquetadora' tendrán prioridad en el orden no-marcado. En español, como en la mayor parte de las lenguas, este principio favorece los nombres propios y desfavorece los nombres comunes, en particular, los de referencia inespecífica.

Como el nivel de especificidad referencial también caracteriza a los elementos fóricos, el principio de Anaforicidad postula que el grado de co-significación es más fuerte en pronombres que en sustantivos, por lo cual en una situación enunciativa con requerimientos deícticos, las unidades pronominales van a ser preferidas frente a los nombres u otros signos transpuestos a la misma categoría (un adjetivo o un infinitivo).

Para concluir, nos referiremos al principio de Complejidad Creciente según el cual las piezas sintácticas son distribuidas sobre la base de su complejidad lexicogramatical. Para Dik (1997: 4II), el citado parámetro distribuye las unidades en una pendiente de complejidad precedida por la unidad más sencilla (el ennegrecido es nuestro):

\footnotetext{
"clitic < pronoun < noun phrase < adpositional phrase < subordinate clause".
} 
Esta jerarquía apoya la tesis pragmática la cual propone que inicio y término de oración son posiciones sensibles a la ejecución de operaciones discursivo-semánticas especiales. En particular, la posición de los clíticos tiene el valor psicolingüístico de facilitar el procesamiento al ser unidades de baja densidad referencial y mínima complejidad morfosintáctica que se ubican en la zona inicial de la oración. El cumplimiento de este postulado demostraría, según el propio Dik (1997: 410), la existencia de: "strategies for relieving the Prefield [-Head-Postfield] of excessive complexity."

En español, la complejidad creciente se manifiesta en la ubicación preferente de los sujetos extensos en posición final, incluso con predicados que seleccionan el arranque como posición no-marcada del sujeto, caso en que se encuentra el verbo 'respaldar':

(15) Respaldan esta reacción los partidos que no se alinearon con el gobierno.

Muy probablemente, la activación del mismo parámetro subyace a la elección de una construcción pasiva perifrástica, incluida en el siguiente ejemplo:

(16) Esta reacción fue respaldada por los partidos que no se alinearon con el gobierno.

Al contrario, una interpretación centrada en el nivel informativo obligaría a proponer que (16) incluye un fenómeno de tematización por el cual la disposición canónica del argumento objeto "esta reacción" es anulada a través de su gramaticalización como sujeto inicial de la pasiva, análisis admisible basados en el principio (GP7).

Sin embargo, no toda construcción pasiva perifrástica puede explicarse como manifestación de focalidad y asumiendo que (I6) no lo es, resulta verosímil la intervención del principio de complejidad creciente, cuya priorización anula otros parámetros (como la animacidad) y determina la elección de una estructura que gramaticaliza a los participantes agentivos como complemento circunstancial y los sitúa al final de la oración, debido a la no simplicidad de la magnitud seleccionada para instanciar el argumento como sujeto.

El análisis de (I6) quedaría incompleto sin destacar que la doble interpretación a que da lugar su fisonomía se debe al carácter pragmáticamente difuso de la estructura. Según Aarts (2007), entre las categorías y construcciones de una lengua existen unidades híbridas, reconocibles por su capacidad para gramaticalizar propiedades lingüísticas e informativas diversas, caso manifiesto en (I6).

En un trabajo dedicado a la articulación gramáticapragmática, analizamos bajo la perspectiva de gramática difusa (fuzzy grammar), las tres variedades de oraciones hendidas (hendidas, pseudohendidas, pseudohendidas inversas) disponibles en español de acuerdo con el nivel de especificidad de sus propiedades pragmagramaticales, verificando una gradiencia en la capacidad de codificar la función focal. En la escala formada por estas construcciones de relieve, la hendida se comporta como realización prototípica (Pinuer, 2009a).

\section{Des/Activación de principios y des/ colocación de los constituyentes oracionales}

Los 12 principios examinados serán a continuación organizados en la Tabla 2 que pretende reflejar la naturaleza específica de cada parámetro, haciendo explícito su fuente de activación (motivación) asociada a la forma prominente de expresión (gramaticalización).

Si bien optamos por una distribución paradigmática, queda claro que los principios operan interrelacionadamente y de forma tal, que incluso en pares sintácticos mínimos (allosentences), subyacen comportamientos diferenciados explicables por la acción de los parámetros activos, según intentaremos demostrar cuando ahondemos en el orden de la oración declarativa. Por último, y dicho en favor de la Tabla 2 , los principios son abstracciones acerca de la articulación del lenguaje con atributos genéricos de base biológica y cultural, por lo que no existe una categorización unívoca de rasgos y una sola manera de presentarlos.

En primer lugar, cada parámetro viene a reafirmar que el enunciado lingüístico es un objeto de alta complejidad si se consideran las opciones de efecto linear que pueden intervenir en su construcción. Por ejemplo, en la fisonomía oracional puede subyacer la decisión de reproducir icónicamente el evento denotado (iconicidad) y facilitar al destinatario el procesamiento informativo (tema inicial) y sintáctico (complejidad creciente). Sin duda, el material lingüístico es, como menos, evidencia de la disponibilidad de un sofisticado sistema de opciones 
Tabla 2. Principios de orden oracional.

\begin{tabular}{|l|l|l|}
\hline \multicolumn{1}{|c|}{ PRINCIPIO } & \multicolumn{1}{c|}{ MOTIVACIÓN } & \multicolumn{1}{c|}{ GRAMATICALIZACIÓN } \\
\hline Iconicidad & Cosmomorfismo & Actor $<$ Acción $<$ Locus \\
\hline Integridad de dominio & Contigüidad funcional & V $<$ Argumentos \\
\hline Estabilidad funcional & Locus funcional & V_ Compls. Args. \\
\hline Relieve pragmático & Predicación informativa & Base $<$ Foco \\
\hline Tema inicial & Marco compartido & Tema $<$ Rema \\
\hline Nuclearidad argumental & Delimitación predicativa & {$[\mathrm{V}+$ Arg. Term.] + Arg } \\
\hline Animacidad & Naturalización & Humano $<$ Inanimado \\
\hline Actancialidad & Jerarquía dialógica & Ira. Pers. $<$ 3ra. Pers. \\
\hline Agentividad & Control referencial & Causante $<$ Causado \\
\hline Definitud & Individuación & NP $<$ NC abstracto \\
\hline Anaforicidad & Correferencialidad & Pron. Personal $<$ NC \\
\hline Complejidad creciente & Costo cognitivo & Clítico $<$ Clsub \\
\hline
\end{tabular}

lexicogramaticales capacitado para codificar un repertorio amplio de necesidades comunicativas.

En segundo lugar, cada principio desarrolla una gradiente interna donde las expresiones de gramaticalización se jerarquizan de acuerdo con el grado de satisfacción del parámetro. Los recursos de adecuación alta tienen prioridad, pues otorgan mayores condiciones de visibilidad al principio y son, por lo mismo, aquellos que mejor explican un determinado orden. Por el contrario, los recursos bajos son distintivamente débiles, pero más rentables en el potencial de codificación dada su menor especificidad.

En tercer lugar, no es plausible suponer la activación aleatoria de principios en razón de su heterogeneidad y funcionamiento jerarquizado, si bien esto último se desarrolla en un grado incierto de control ya que el hablante no posee acceso pleno al sistema lingüístico. Consideramos pertinente suponer cierto nivel de regulación en aquellos orientados al marco dialógico (relieve pragmático, animacidad, agentividad) y no a otros (estabilidad funcional, anaforicidad, complejidad creciente) que, regulados por el sistema, se comportan por 'efecto en cadena' para facilitar el despliegue de los principios activados.

Nuestra exposición e ilustración se ha circunscrito a las características de una lengua flexiva y analítica como el español. Sin embargo, y por lo obvio solo haremos mención de ello, las lenguas, de acuerdo con su tipología general y tendencias evolutivas, manifiestan heterogéneamente la intermediación de los parámetros. En español, la posición inicial y final de oración son muy productivas para introducir relieve informativo, operación que también puede ser ejecutada a través de un morfema o un acento en una lengua donde el orden no sea una propiedad relevante. Recordemos que los principios no tienen apariencia lexicogramatical, aunque la forma lingüística sea su modo de expresión natural.

En lenguas de orden flexible, la disposición de los constituyentes oracionales hace irrefutable la participación de los principios lineares en el trazado sintáctico. Como se indicó antes, la colocación sintagmática muestra depender del control activo de los parámetros jerarquizados de mayor prominencia, aunque el proceso de desactivación difícilmente pueda ocurrir en las mismas condiciones de atención. En definitiva, todo orden oracional se sustenta en una priorización de estos principios, fenómeno que retomaremos a partir de (I7) con la intensión de profundizar en su funcionamiento:

(17) Los vecinos la presentaron al concejo municipal. (cfr. (2))

La selección del clítico implica la desactivación de la integridad de dominio y la estabilidad funcional al hacerse prominente la anaforicidad por un requerimiento (supuesto aqui) de cohesión discursiva que aproxima la unidad fórica a su antecedente ('la demanda'). Para ponderar la anulación de ambos principios sirve recordar que en el español medieval los referentes pronominales dispusieron de una flexibilidad sintagmática similar a la de un sintagma nominal, que después perdieron cuando se fijó su carácter clítico y función correferencial. 
Por su parte, la dislocación a la izquierda también visibiliza una serie de implicancias en la operatividad de los principios y sus escalas:

\section{(I8) La apelación la presentaron los vecinos al concejo municipal.}

En este ejemplo, la relevancia pragmática (GP7) se impone a la iconicidad, animacidad, estabilidad funcional e integridad de dominio. Los dos primeros habrían ubicado el sintagma de referencia animada en posición inicial, en tanto que los principios restantes situarían el sintagma nominal objeto después del verbo. La jerarquización de la relevancia es fundamental para ubicar el $\mathrm{OD}$ en posición de sujeto, locación donde pasa a operar como material informativo dado, aunque esta explicación no debe generalizarse, ya que la codificación del complemento objeto como tema o tópico de oración es una estrategia que no tiene a la colocación lineal como dispositivo único de gramaticalización. Además, funciones como tópico, foco y el propio tema deben entenderse como valores informativos de la oración, con independencia a: "the structural positions in which they may be manifested" (Van Valin, 2005: 68).

Otro efecto del parámetro de relieve es la determinación del orden marcado de (I8), rasgo que determina su fuerte dependencia del contexto, el cual provee las condiciones informativas de interpretación. Entre más pragmática es una entidad, más específicas y restringidas son sus apariciones. Todo esto nos anima a plantear que la subdeterminación contextual es muy posiblemente la principal causa del constreñimiento que muestran estas construcciones cuando se comparan con sus equivalentes no afectadas o neutras. Por ejemplo, la oración (18) solo puede ser respuesta aceptable a la pregunta: '¿dónde presentaron los vecinos la apelación?' En cambio, (2) tiene la posibilidad de seleccionarse como respuesta apropiada para las preguntas: ‘qué pasó?', ‘qué hicieron los vecinos?', ‘qué presentaron los vecinos?', ‘dónde presentaron los vecinos la apelación?'

Con pocas excepciones, la ejemplificación examinada hace patente la sintaxis flexible del español, resultando contraintuitivo negar que sea, por lo mismo, una lengua de orden libre. Pero en rigor se trata de un orden flexible, en el cual factores de índole no-estructural desempeñan un papel clave en la distribución sintagmática. En suma, la forma linear depende tanto de factores informativos como de principios relacionados con la naturaleza de los participantes en el evento comunicativo ( \pm animado) y a la semántica (e.gr. capacidad de definitud) y complejidad sintáctica de las estructuras lexicogramaticales desplegadas.

Los principios interactúan en una lógica de complementación y competencia, razón por la cual no se atribuye a ninguno de ellos el estatus de principio superior. Sin embargo, se aprecia, a pesar de la limitada muestra descrita, que el relieve exhibe mayor capacidad para anular otros principios o, mejor todavía, una cobertura más amplia para afectar la operatividad de los restantes parámetros.

Esta interpretación encuentra asidero en la correlación inversa entre el nivel de flexibilidad distribucional y el impacto de la función focal. En efecto, de acuerdo con Van Valin y LaPolla (2004: 4I8):

"In languages with very fixed word order, focus structure has little or no direct effect on word order [...] In languages with more flexible word order [...] the impact of focus structure can be very great."

Cabe destacar que en el modelo generativo también se reconoce la implicancia del factor informativo a partir de la capacidad del constituyente focal para fijar su posición en el enunciado:

"La presuposición precede al foco o, dicho de otra manera, el foco es el constituyente que aparece «a la derecha» estructuralmente" (Bosque \& Gutiérrez-Rexach, 2009: 680).

Es común que la sintaxis flexible del español sea contrapuesta a la rigidez de la sintaxis inglesa (Erteschik-Shir, 2007) e inversamente, contrastar la plasticidad del inglés para asignar el acento oracional. Una muestra de la limitada flexibilidad de la acentuación oracional española se encuentra en la existencia de posiciones prosódicas oportunas:

(19) Una propuesta entregaron al candidato presidencial [los vecinos $\left.{ }_{\mathrm{Foco}}\right]$

La dislocación a la derecha del $\mathrm{SN}$ sujeto está determinada por una convención distribucional del acento nuclear neutro: "se coloca en la última palabra del grupo (o constituyente) melódico” (Zubizarreta, 
1999: 4229), es decir, en la última palabra delante de un punto, punto y coma o coma. Consecuencia de lo anterior, el llamado foco neutro debe situarse en la última posición del grupo prosódico, fenómeno característico, por otra parte, de las lenguas de predicación inicial (Van Valin \& LaPolla, 2004), como el español.

Para Rodríguez (2005), las dislocaciones son 'movimientos prosódicos' que permiten llevar o sacar un constituyente de un determinado dominio acentual. La autora distingue entre desplazamientos de focalización y defocalización. En (I9), el segundo sería responsable de la ubicación inicial del SN objeto, el cual se encuentra en posición defocalizada; el primero, en cambio, llevaría el SN sujeto al dominio del acento focal, lugar donde el segmento recibe prominencia acústica y adquiere el estatus informativo de foco neutro.

La adecuación empírica de los denominados 'movimientos prosódicos' asigna al planteamiento de Rodríguez (2005) un alto valor explicativo para los fenómenos de distribución marcada, sin embargo, al sugerir que focalización y defocalización son operaciones que mueven constituyentes, contradice el principio funcional de que las reglas que explican el orden de palabras capturan parámetros de colocación. Con todo, esta solución presenta importantes coincidencias con la postura dikeana, ante lo cual no puede descartarse la posibilidad de enriquecer el análisis con la referida tesis. En efecto, una y otra formulación coinciden en dos asunciones importantes: la primera, que las lenguas disponen de un orden básico; y la segunda, que las variaciones de ese orden afectan las posiciones de mayor preeminencia cognitiva y pragmática.

En definitiva, interpretaremos el movimiento prosódico como 'colocación prosódica' y factor de expresión lingüística que gramaticaliza elecciones semántico-discursivas, de acuerdo con lo cual OVS no es movimiento de SVO sino una alternativa que, activada por el principio de relieve, habilita la función sujeto para recibir el acento nuclear neutro. Al contrario, la 'deslocación prosódica' determina una posición 'inoportuna' que anula la potencialidad de recibir el incremento acústico y manifestar focalidad que, dicho sea de paso, además de la función propiamente focal, incluye el énfasis y la intensificación (Pinuer, 2009b).
Para confirmar el poder explicativo de la 'colocación prosódica' como factor de relieve, examinaremos una construcción hendida o copulativa enfática (RAE, 2009) que acompañamos con un análisis informativo tomado de Butler (2005):

(20) Fueron los vecinos quienes entregaron una propuesta al candidato presidencial
Tema Rema
$\leftarrow$ Tema $\rightarrow$

El análisis distingue la estructura informativa de la oración en términos de la distribución de lo conocido (tema) y lo novedoso (rema), plano en que corresponde agregar la presencia de un segmento focal o epicentro referencial, 'los vecinos', que se comporta como la variable de más alto valor informativo (foco). Por su ubicación inicial, se puede inferir que se trata de un foco de contraste, pues ocupa la posición acentual oportuna. Si estuviera en posición final (quienes entregaron una propuesta al candidato presidencial fueron los vecinos), sería un foco neutro y la construcción, una oración pseudohendida.

\section{CONCLUSIONES}

Primeramente, destacaremos que la importancia y cobertura disciplinaria recibida por el orden de palabras se explica por tres hechos de gran impacto heurístico. El primero es que opera como un parámetro muy productivo en los estudios tipológicos, sobre todo cuando no se limita a la identificación de categorías y funciones. En segundo lugar, el orden de constituyentes ha mostrado ser una variable de notorio rendimiento en la búsqueda de evidencia psicolingüística relacionada con la adquisición y procesamiento del lenguaje. Seguidamente, manifiesta con claridad la empatía de los hablantes hacia una serie de principios que determinan la fisonomía lingüística e informativa de sus mensajes.

Si bien SVO no forma parte de los universales lingüísticos, es el trazado básico, condición respaldada en evidencia tipológica, cognitiva y discursiva. Los datos disponibles permiten confirmar asunciones tan importantes como: ser la distribución no-marcada en la mayor parte de las lenguas, el taxema de adquisición más temprana y de menor costo de procesamiento, y estrategia informativa orientada a incrementar la comprensión en el destinatario. Luego, no puede sorprender que SVO haya sido considerado 'orden ubicuo' o 'fósil sintáctico'. 
Hoy por hoy, la mayoría de los principios lineares son epistemológicamente transversales, pues se emplean en investigaciones sustentadas en diversos marcos disciplinarios y perspectivas teóricas, eso sí, sobre la base de dos visiones contrapuestas para entender su naturaleza explicativa. Una, fundamentalmente generativa, los vincula a operaciones sintácticas de estructura profunda; otra, esencialmente funcional, los conceptualiza como reglas de colocación. En nuestro caso, seguimos este último enfoque porque adherimos a la hipótesis de que la disposición de constituyentes codifica parte del significado referencial e informativo de la oración, por lo que las variaciones posicionales de unas mismas unidades deben interpretarse como la gramaticalización de enunciados distintos.

Aun cuando la presentación no incluyó datos cuantitativos, de todas formas es posible plantear ciertas generalizaciones extraídas del comportamiento de la oración di/transitiva en español y que enunciaremos en grado ascendente de importancia: (i) todos los parámetros manifiestan algún nivel de activación; (ii) los principios de agentividad y complejidad creciente se perfilan como parámetros fundamentales; (iii) el principio de relieve muestra ser la noción con mayor capacidad de interferencia en el despliegue de los restantes parámetros.

Una de las variables ausentes en este trabajo fue la noción de género, de ahí que no podemos dimensionar cuál(es) y/o cuánto(s) de los resultados podrían estar mediatizados por el formato textual. Al respecto, nuestra impresión es que el fenómeno linear puede constituir un rasgo singularizador si se empalma con la dimensión enunciativa, en particular, con el propósito discursivo (informar/influir) y el rol asignado al destinatario (iniciado/experto).

En suma, el orden de constituyentes oracionales se ofrece como uno de esos problemas cuya discusión pone de manifiesto que teorizaciones construidas con diferentes enfoques epistemológicos son igualmente válidas. Puede admitirse que el orden es lo que cada teoría plantea, de modo que un modelo explicativo debe incorporar diferentes aristas del fenómeno y explicar su interacción. 


\section{REFERENCIAS BIBLIOGRÁFICAS}

Aarts, B. (2007). Syntactic gradience. The nature of grammatical indeterminacy. Oxford \& Nueva York: OUP.

Alonso-Cortés, Á. (2008). Lingüística. Madrid: Cátedra.

Bosque, I. \& Gutiérrez-Rexach, J. (2009). Fundamentos de sintaxis formal. Madrid:Akal.

Branigan, H., Pickering, M. \& Tanaka, M. (2008). Contributions of animacy to grammatical function assignment and word order during production. Lingua, I I8, I 72-I89.

Butler, C. (2005). Focusing on focus: A comparison of functional grammar, role and reference grammar and systemic functional grammar. Language Sciences, 27, 585-6I8.

Butler, C., Mairal, R., Martín, J. \& Ruiz, F. (1999). Nuevas perspectivas en gramática funcional. Madrid:Ariel.

Casado, P., Martín-Loeches, M., Muñoz, F. \& Fernández-Frías, C. (2005). Are semantic and syntactic cues inducing the same processes in the identification of word order? Cognitive Brain Research, 24, 526-543.

Dik, S. (1997). The theory of functional grammar. Part I:The structure of the clause. Berlin \& Nueva York: Mouton de Gruyter.

Eguren, L. \& Fernández, O. (2004). Introducción a una sintaxis minimista. Madrid: Gredos.

Erteschik-Shir, N. (2007). Information structure. Cambridge \& Nueva York: OUP.

Escandell, M. (2004). Fundamentos de semántica composicional. Barcelona:Ariel.

Godeo, M. (2000). Orden de palabras y categorización lingüística. Revista Española de Lingüística Aplicada, Volumen Monográfico, 395-408.

Greenberg, J. (1963). Some universals of grammar with particular reference to the order of meaningful elements. Universals of Language, 73-I I3.

Halliday, M. A. K. (1978). Language as social semiotic. The social interpretation of language and meaning. Londres: Edward Arnold.

Halliday, M. A. K. (2005). On grammar. En J.Wenster (Ed.), Collected Works of M.A.K. Halliday (Vol. I). Londres: Continuum.

Heine, B. \& König, C. (2008). On the linear order of ditransitive objects. Language Sciences, doi:I0.1016/j.langsci.2008.07.002.

Hengeveld, K., Rijkhoff, J. \& Siewierska, A. (2004). Parts-of-speech systems and word order, Journal of Linguistics, 40(2), 527-570.

Hengeveld, K. \& Mackenzie, J. (2008). Functional discourse grammar.A typologically-based theory of language structure. Londres \& Nueva York: OUP.

Lambrecht, K. (1998). Information structure and sentence form. Topic, focus and the mental representations of discourse referents. Cambridge \& Nueva York: CUP.

Lehmann,W. (1973).A structural principle of language and its implications. Language, 49, 47-66.

Martínez, E. (1999). Gramática del discurso: Foco y énfasis en inglés y en español. Barcelona: Promociones y Publicaciones Universitarias.

Padilla, X. (200I). El orden de palabras en el español coloquial. Tesis Doctoral, Universitat de Valencia, España.

Padilla, X. (2005). Pragmática del orden de palabras. Valencia: Ediciones Universidad de Alicante. 
Pinuer, C. (2009a). La función focal en la gramática: Implicancias teóricas y repercusiones metodológicas. ALED, Revista Latinoamericana de Estudios del Discurso, 9(I), I I 3- I 34.

Pinuer, C. (2009b). La dimensión de focalidad: Conceptualización, instanciación y taxonomías. Revista Signos. Estudios de Lingüística, 42(69), 83-106.

Rappaport, M., Doron, E. \& Siche, I. (20 I0). Lexical semantics, syntax, and event structure. Londres \& Nueva York: OUP.

Real Academia Española (RAE). (2009). Nueva gramática de la lengua española, Vol. II. Madrid: Espasa Libros.

Rodríguez, T. (2005). Manual de sintaxis del español. Madrid: Castalia.

Sánchez, M. (2004). Orden básico y órdenes marcados en español. En G. Solís (Ed.), Cuestiones de lingüística general e hispánica (pp. 259-285). Lima: Universidad Nacional Mayor de San Marcos.

Van Valin, R. (2005). Exploring the syntax-semantics interface. Cambridge \& Nueva York: CUP.

Van Valin, R. \& LaPolla, R. (2004). Syntax. Structure, meaning and function. Cambridge \& Nueva York: OUP.

Vennemann, T. (1974). Topic, subject and word-order: From SXV to SVX via TVX. En J. M. Anderson \& C. Jones (Eds.), Historical linguistics (pp. 339-376). Amsterdam: NorthHolland.

Zubizarreta, M. (1999). Las funciones informativas: Tema y foco. En I. Bosque \& V. Demonte (Coords.), Gramática descriptiva de la lengua española (pp. 42 I5-4246). Madrid: Espasa Calpe.

\section{NOTAS}

' En este periodo el fenómeno se estudió bajo diversas denominaciones, de las cuales tomaremos unas ('patrón sintáctico', 'taxema', 'configuración oracional', 'fisonomía linear') y prescindiremos de otras ('linearización' y 'alineación') por su dudosa equivalencia.
${ }^{2}$ La noción de cotejo de rasgos es una de las varias y valiosas recomendaciones recibidas del per review. Agradecemos la lectura crítica y propositiva, en virtud de la cual hemos introducido importantes mejoras al artículo.

* Trabajo desarrollado en el marco de Proyecto FONDECYT I090464, Comisión Nacional de Investigación Científica y Tecnológica, Chile. 